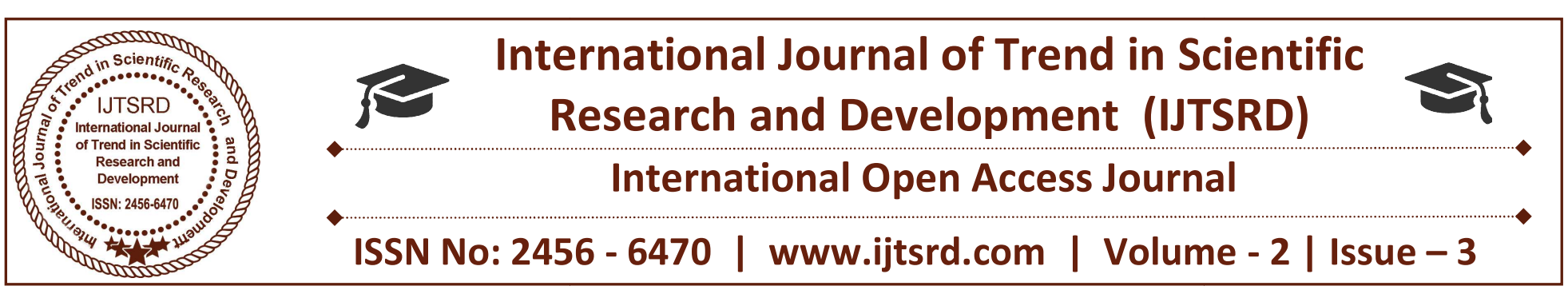

\title{
Business Excellence Model: A Selective Study
}

\author{
Thenmozhi Arukutty \\ M Phil, Dr. SNS Rajalakshmi College of Arts and Science, \\ Coimbatore, Tamil Nadu, India
}

\begin{abstract}
Business today is being impacted by multiple forces - economic shocks, atomization of markets and demand, borderless commerce, advances in technology, a sense of hastening, and deconstruction of business. This paper starts the argumentation from how BEMs are dichotomously perceived by adopters, either prescriptive or descriptive. The prescriptive aspect indicates that the adopters treat BEM as a 'must' to achieve business excellence. And the descriptive aspect indicates that the adopters treat $\mathrm{BEM}$ as a 'reference' to inspect how the BE of the enterprise is. An effective BEM should be prescriptive-based for the principle of providing a 'total solution' to organizational management. To be this, the deficiencies found in the existing BEMs are firstly reviewed and the arguments in relation to the deficiencies are summarized. Then, four fundamental grounds for a inclusive BEM are raised in response to those deficiencies. A comprehensive model to meet the fundamental premises is finally proposed.
\end{abstract}

Keywords: Business Excellence, Total Quality Management, Self-assessment, Change Management

\section{INTRODUCTION}

Business Excellence (BE) is about developing and strengthening the management systems and processes of an organization to improve performance and create value for stakeholders. $\mathrm{BE}$ is much more than having a quality system in place. $\mathrm{BE}$ is about achieving excellence in everything that an organization does (including leadership, strategy, customer focus, information management, people and processes) and most importantly achieving superior business results. Some of the tools used are the balanced scorecard,

Lean, the Six Sigma statistical tools, process management, and project management. As described by the European Foundation for Quality Management (EFQM), BE refers to exceptional practices in managing the business and achieving results in terms of a set of eight fundamental concepts. These concepts are 'results orientation, customer focus, leadership and constancy of purpose, management by processes and facts, people development and involvement, continuous learning, innovation \& improvement; partnership development, and public responsibility.'

Besides, business excellence models (BEMs) have been generally developed by national bodies as a basis for award programs. For most of these bodies, the awards themselves are secondary in importance to the widespread adoption of the concepts of business excellence, which ultimately leads to improved national economic performance. By far the majority of organizations that use these models do so for selfassessment, through which they may identify improvement opportunities, areas of strength, and ideas for future organizational development. Users of the EQA Excellence Model, for instance, do so for the following purposes: self-assessment, strategy formulation, visioning, project management, supplier management, and mergers. The most popular and influential model in the western world is the Malcolm Baldrige National Quality Award Model (also known as MBNQA model, the MBNQA Criteria, or the Criteria for Performance Excellence), launched by the US government. More than 60 national and state/regional awards base their frameworks upon the Baldrige criteria. 
Due to BEMs have been widely implemented in the industries over two decades, a ton of experiences and evidences are accumulated. Researches were conducted to investigate their effectiveness which includes the benefits and the deficiencies. Some researches indicated that organizations obtained significant benefits. The benefits include the financial profit and the non-financial outcomes. However, it has been noted that not all the messages are positive. On the one hand, some research findings pointed out that an excellence approach is not a guarantee of success. Also, although there is compelling evidence that business excellence delivers benefit to the organization, it is clear that it does not work for everyone. On the other hand, practitioners usually complain that they encounter difficulties in implementing BEM which include sophisticated assessment criteria, lack of infrastructure, quality bureaucracy, excessive paperwork, cumbersome procedures, time consuming, and lack of focus. Why the research findings are inconsistent? Why the practitioners meet difficulties? To answer these questions is not an easy task. It's a complicated issue in nature, which involves many contingency factors in the implementation of BEMs, such as size, industrial sector, organizational structure $O$ \& $/$ system infrastructure, culture, and the degree of quality maturity, etc.

The perception of BEMs will guide their behaviors of using the management tool. In the way of dichotomy, BEMs can be perceived either prescriptive or descriptive. The use of BEM is mainly for the purposes of confirming if they have already fulfilled the requirement of the quality award. The present instigator holds that the BEM should be prescriptivebased for the purpose of providing a 'total solution' to every aspect of organizational management. However, the past literature indicated that it is still under debating for whether the existing BEMs (such as MBNQA, EQA, etc) are prescriptive or not. Based on the reasons stated above, to develop a rather comprehensive BEM which is able to help an organization in pursuit of business excellence becomes necessary. More specifically, in this paper, the present instigator intend to achieve the following research objectives: (1) to give an introduction to the existing BEMs and self-assessment, (2) to summarize the deficiencies of the existing models, (3) to raise the fundamental premises for a comprehensive business excellence model, and finally (4) to propose a comprehensive model to meet the fundamental premises.

\section{LITERATURE REVIEW}

\subsection{BEMs and Self-assessment}

BEM is not only designed to present the criteria and procedures to compete award winner; its purpose is to become an effective self-assessment tool for those who are interested in quality and allocate recourses to serve as guidance for improving their organizations. That is to say, the model is geared not only to the organizations in a position to successfully compete for the award but also to those who wish to take up the challenge of pursuing competitiveness and business excellence. Porter \& Tanner (2004, p287-312) proposed an eight-step common processes for an organization to conduct a self-assessment (see Figure 1). It starts from choosing the framework and ends up with eliciting the action plans for those that are necessary to be corrected or improved. (Choosing the framework, Forming the assessment team, Collecting the information, Assessment and scoring, Consensus, The site visit process - clarification and verification, Feedback, Action planning)

\subsection{The Extension Models}

Despite BEMs presented above are designed to serve as a total solution of business management, it is still experienced by practitioners the necessity of further guidance in the implementation. The extension models are then developed to remedy the problems. Some of the models are self-assessment roadmap, self-assessment methodology, self-assessment decision model and and 4P quality strategy model etc,. Among them, it is interesting to find that each model was proposed to respond certain specific deficiencies respectively. Porter \& Tanner in 2004 proposed self-assessment roadmap is in response of the general defined assessment criteria. This extension model is a contingency-based, which the roadmap is divided into three phases: 'entry', 'user' and 'worldclass' in terms of the experience of practicing selfassessment. On the other hand, 'self-assessment methodology' and 'self-assessment decision model' are proposed to respond the criticism on measurement system.

4P quality strategy model was proposed by Dahlgaard \& Dahlgaard-Park in the early 90s. The '4P' definition is based on the argument that the first 
priority of any quality or excellence strategy should be to build quality into 'people' as the essential foundation and catalyst for improving 'partnerships', 'process' and 'products'. The aim of quantitative self-assessment process is to involve everyone in improving enablers as well as results. The 'vital few' improvement areas are then identified by choosing the biggest differences between the two measures. The organizations finally establish cross-functional improvement team to come up with action plans by using quality map. This model was adopted firstly by the Post Denmark in 1990 and used by several Scandinavian companies after that time. In the opinion of the authors, this model is applicable in almost any organization to ensure a successful start up in TQM, or excellence building process.

\subsection{The Benefits and the Deficiencies}

The positive and supportive research can be summarized into two categories. One of the two categories is to directly investigate the business results for those BEM adopters. The business results include the financial indicators and the non-financial indicators. The other category is to investigate the cause-effect linkages among the enablers and the business results.

The other researches point out the deficiencies which are related to the operations of self-assessment or award application. The operational deficiencies include excessive paperwork, cumbersome procedures, time consuming and quality bureaucracy, etc. To meet the award's requirements it is necessary to collect vast amounts of internal and external information, analysis the data and expend substantial amounts of managerial effort.

\subsection{Arguments in response to the Deficiencies}

- The first argument is that the existing models are rarely made adjustments in accordance with the scientific empirical evidences. It would result in the models deviating from the practical arena and the user-friendly perspective.

- The second argument is that the existing models, except self-assessment roadmap and MBNQA, do not include the contingency factors which are particularly important to the organization with different size, industry sectors and experience in practicing self-assessment.

- The third argument is that the existing models, except 4P quality strategy, are essentially attributed to a sort of assessment tools only instead of serving as a 'total solution' management tool.

- The fourth argument is that the existing models have not had enough persuasiveness to convince 'total employee involvement' which is 'the must' in self assessment.

- The fifth argument is that the existing models are lack of integration in the operational level in spite of they do have the holistic view in conceptual level.

\section{FUNDAMENTAL PREMISES FOR A COMPREHENSIVE BEM}

Having the review of the deficiencies in relation to the existing models, we turn out the way to postulate four fundamental premises (FPs) which are, on the one hand, in response of the arguments raised in the pervious section and, on the other hand, to highlight the required principles for the new model that will be proposed in the next section.

FP 1: It should be a prescriptive-based model but make adjustments in accordance with the empirical evidences which are descriptively derived from the scientific research.

Quality is commonly measured by two dominant factors, 'functions' and 'cost'. It can be mapped to a good quality management tool which is measured by 'usefulness' and 'easy-of-use'. It is apparent that a good quality BEM should be embedded the two dominant factors. 'Usefulness' means that adopters choose this management tool in the beginning, and the excellent outcomes are finally achieved through following the guidance of the management tool. 'Easy-of-use' means that the management tool is designed by fitting the propensity of humankind, and less mental effort or attention is necessary to pay on it.

FP 2: It should work well not only as a measurement model but also as an organizational improvement model in the self-assessment or the quality award competition.

As we know, BEMs are designed not only to provide the criteria and procedures for the competition of quality award but also the main purpose is to become an effective self-assessment tool for those who are interested in quality management and generate the action plans to improve their organizations. However, the empirical experiences show that both functions often do not exist in the same platform or an organization. 
FP 3: It should be not only seamlessly merged into the existing system in an organization but also totally accepted by employee.

It is no doubt that the BEM initiatives are utilized for the assistance of an organization in achieving business excellence and organizational sustainability. To accomplish the objective, it is no way without the total acceptance and getting BEM into a habit of daily operation by employee. In practice, integration of the BEM into the existing system can be achieved through a combination of multiple management activities in the organization, including using it as part of the strategic planning process, aligning with other organizational systems, linking with performance management and involving staff in it through teamwork. However, it is found that a number of issues and problems are associated with the implementation, such as excessive paperwork, cumbersome procedures, time consuming and quality bureaucracy, etc., which were mentioned above. These operational deficiencies always keep practitioners away for this management tool. In our opinions, it is resulted from too little focusing on understanding the human factor.

FP 4: It should provide a ready-to-use guidance to incorporate with the other Omanagement tools/techniques.

In subsequence of the argumentation in FP2, the existing BEMs are recognized as a good tool in conducting the assessment but it is still deficient in facilitating the organizational improvement. To be a total solution, it is necessary to incorporate the other management approaches into BEMs to remedy this deficiency

\section{DEVELOPMENT OF A COMPREHENSIVE BEM: FACT}

The proposed model is a three-dimensional design which is called 'FACT'. 'FACT' indicates the abbreviation of 'Framework of BE', 'Add', 'organizational Culture/Characteristics' and 'management Tools/Techniques'. It means that a comprehensive BEM should integrate with not only using $\mathrm{BE}$ framework to be guidance and to make assessment of an organization, but also choosing the appropriate management tools/techniques and cultivating the right organizational culture/characteristics towards BE.

\subsection{Framework of BE}

First of all, the use of BE framework is for two purposes: guiding the organization towards $\mathrm{BE}$ and conducting assessment of the performance. Guiding towards BE is the primary purpose for the intension of pursing organizational sustainability. And, conducting assessment is the secondary purpose which can be either self-assessment to diagnose the strength/weakness of an organization or award examination to select the winners.

\subsection{Management tools/techniques}

The use of management tools/techniques is one of the three major dimensions in FACT. Three types of management tools/characteristics are categorized in this study: planning, operation and improvement. Each category has its specific function. The tools used for the function of planning include SWOT, Ho-Shin strategic management, Balance Score Card (BSC), Segmentation-Targeting-Positioning (STP), etc. The tools used for the function of operation include $5 \mathrm{~S}$, Supply Chain Management (SCM), Enterprise Resource Planning (ERP), Just-in-Time (JIT)/Toyota Production System (TPS), Project Management (PM), Total Preventive Maintenance (TPM), Knowledge Management (KM), etc. And the tools used for the function of improvement include Quality Control Circle (QCC), 6 $\sigma$, Business Process Reengineering (BPR), Benchmarking Management (BM), Change Management (CM), etc.

\subsection{Organizational culture/characteristics}

Cultivating the right organizational culture/characteristics for an organization towards BE is the third major dimension in FACT. It is placed in the outer circle to surround the other two dimensions. It implies that it is impossible to have a successful BE without the right organizational culture/characteristics. Two issues are raised here: one is what the right organizational culture/characteristics is, and the other is how it can be cultivated. In response of the first issue, the present authors postulate that 'integration' and 'total involvement in BE' are the two most important culture/characteristics in FACT which ought to be cultivated in an organization. In response of the second issue, how the organizational culture/characteristics can be cultivated in an organization, the present authors suggested the theory proposed by Hofstede et al. (1990). Hofstede et al. classified manifestations of culture into four categories which are 'symbols', 'heroes', 'rituals' and 'values'. 


\section{IMPLICATIONS AND CONCLUSIONS}

\subsection{Implications of FACT}

Having the development of FACT in the previous section, the implications of the model are further illustrated in the following. Instead of using 'Chinese Ta Chi' as the metaphor of the fusion between both dimensions of $\mathrm{BE}$ framework and Management tools/techniques in an organizational management, the trinity is used to implicate the three dimensions of FACT as a whole. Figure 3 present the illustrations of the trinity of the model.

The idea of using the trinity as the implications of FACT is derived from the Christian doctrine. This term has been stated in Christian theology since the beginning of the third century. The word of trinity in etymology is derived from Latin 'trinitas' which

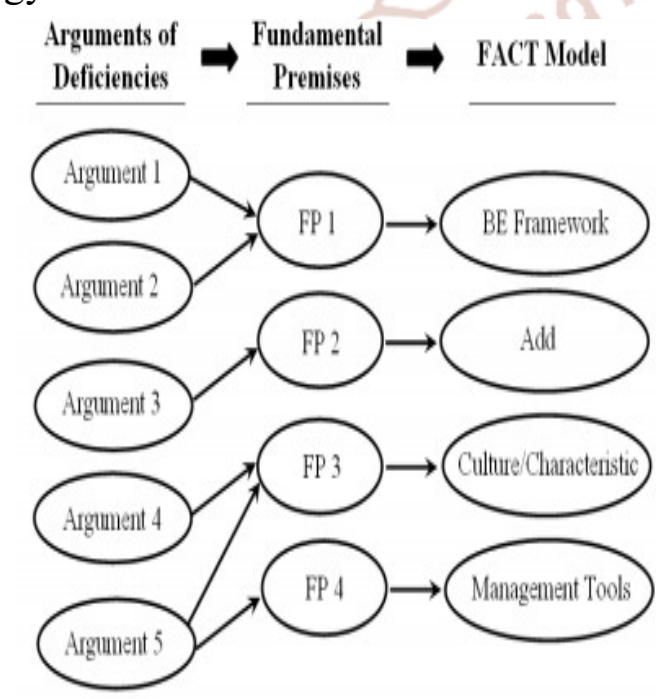

Figure 5 Mappings of deficiency arguments, fundamental premises and FACT means 'the number three, a triad'. The corresponding word in Greek, 'tpiás', means 'a set of three'. In this

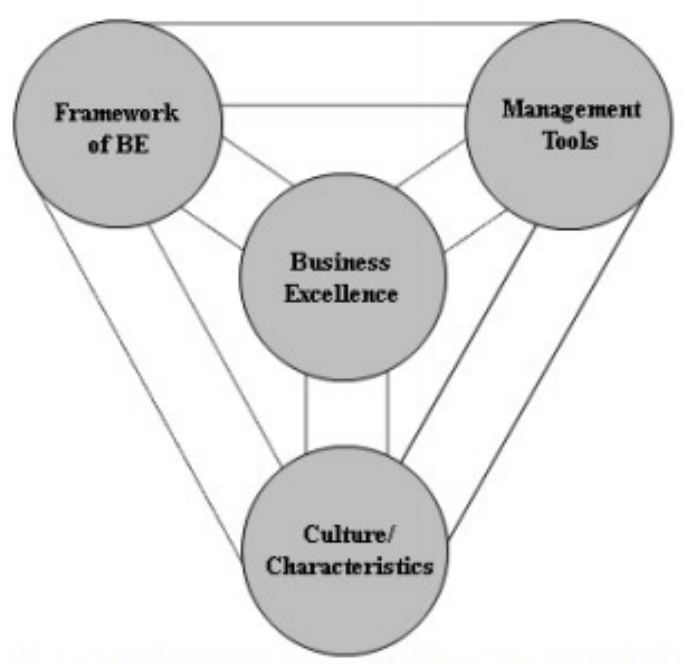

Figure 3 Illustration (I) of the trinity of FACT paper, the correspondence with the trinity in etymology of Latin and Greek can be referred to the three dimensions in FACT come together as one goal towards BE. It indicates that not only the fusion of BE framework and management tools/techniques are the two 'must' in the implementation of BEM but also the cultivation of right organizational culture/characteristics is necessary of the third 'must' as well (see Figure 3).

\subsection{Mappings of deficiency arguments, fundamental premises and FACT}

Figure 5 presents the mappings of deficiency arguments, fundamental premises and FACT. The purpose of the mappings is to examine if the new model proposed in this paper does respond all deficiencies found in the existing BEMs. The mappings are individually illustrated in the following. The first two arguments are criticized in regard to the lacks of scientific evidences and contingency factors in the existing model designs. The present authors argue that a new model should be prescriptive-based in order to be not only the guidance of users but also the measurement standard in an organization. This is the point to be postulated in FP1 and the dimension of 'BE framework' in FACT model. The third argument is criticized in regard to BEM is basically recognized as a measurement tool instead of the provision of a total solution. The present authors hold that a new model should be functioned as not only the measurement but also the planning/operation/improvement in an organization. This is the point to be postulated in FP2 and the necessity of 'add' more dimensions in the new model. The fourth and the fifth arguments are criticized in regard to the lacks of persuasiveness for total involvement and integration in the operational level. The present authors hold that a new model should be easily integrated with not only the systems that have been existed but also the management tools that are appropriate in an organization. These are the points to be postulated in FP3, FP4 and the dimensions of 'organizational culture/characteristics' \& 'management tools/techniques' in FACT model.

\subsection{The possible future research}

The development of a comprehensive BEM is set for the objective in the beginning of this research. A three-dimensional BEM is finally proposed which is called 'FACT'. The contribution of this paper is significant, however it is the first study of this proposed model, some issues are planned for the 
further research. The empirical case studies will be first conducted to investigate, in term of FACT model, what the journey of a BE enterprise had been through. This is for the purpose of developing the roadmap of BE. Here, different forms of organizations can be included as the subjects of the empirical case studies, such as independent enterprise, enterprise group, enterprise supply-chain system or even industrial sector. Upon enough number of the empirical case studies being accumulated, the research objective will be focus on the improvement of national economic performance which is to investigate the benefits of BEMs from the macro viewpoint.

\section{REFERENCES}

1. Ahmed, A.M., Yang, J.B. \& Dale, B.G. (2003) "Self-assessment methodology: Theroute to business excellence", Quality Management Journal, 10(1), 43-57.

2. Caudron, S.W. (1993) "Change keeps TQM programs thriving", Personal Journal, 72 (10), 104-107.
3. Chapman, C. (2000) "The public and private faces of excellence", Quality World, 26(5), 30-32.

4. Collins, J. (2001) Good to Great: Why Some Companies Make The Leap...And Others Don't. , Harper Business, New York.

5. Conti, T. (1997) Organizational Self-assessment, Chapman \& Hall, London.

6. Conti, T. (2002) "A road map through the fog of quality and organizational assessments", Total Quality Management \& Business Excellence, 13(8), 1057-1068.

7. Conti, T. (2007) "A history and review of the European Quality Award Model", The TQM Magazine, 19(2), 112-128.

8. Chin, K.S. \& Pun, K.F. (2002) “A proposed framework for implementing TQM in Chinese organizations", International Journal of Quality \& Reliability Management, 19(3), 272-294.

9. Dale, B.G., van der Wiele, T., Williams, R. \& Greatbanks, R. (1998) "TQM the challenges for European business", Quality World, 46-49. 